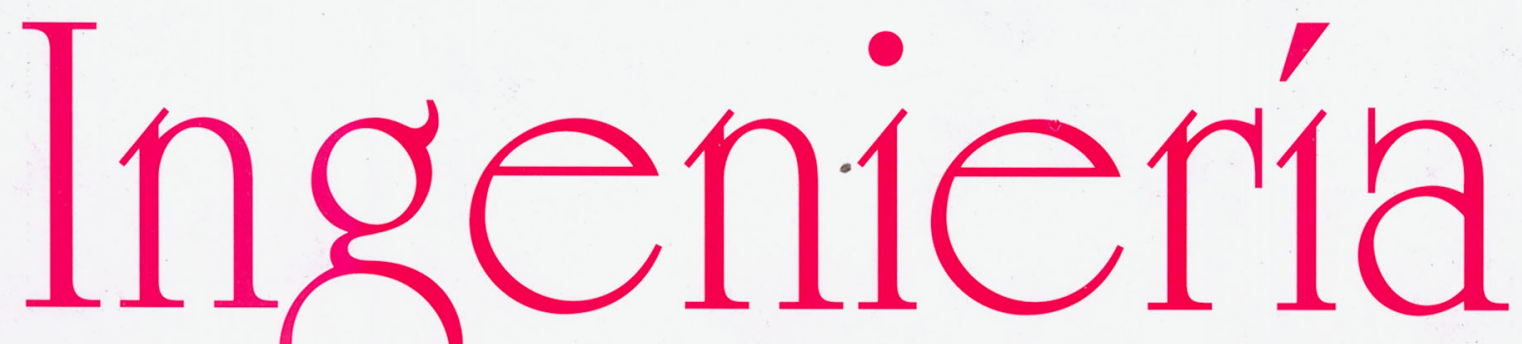

Revista de la Universidad de Costa Rica Enero/Junio 1996 VOLUMEN 6 № 1
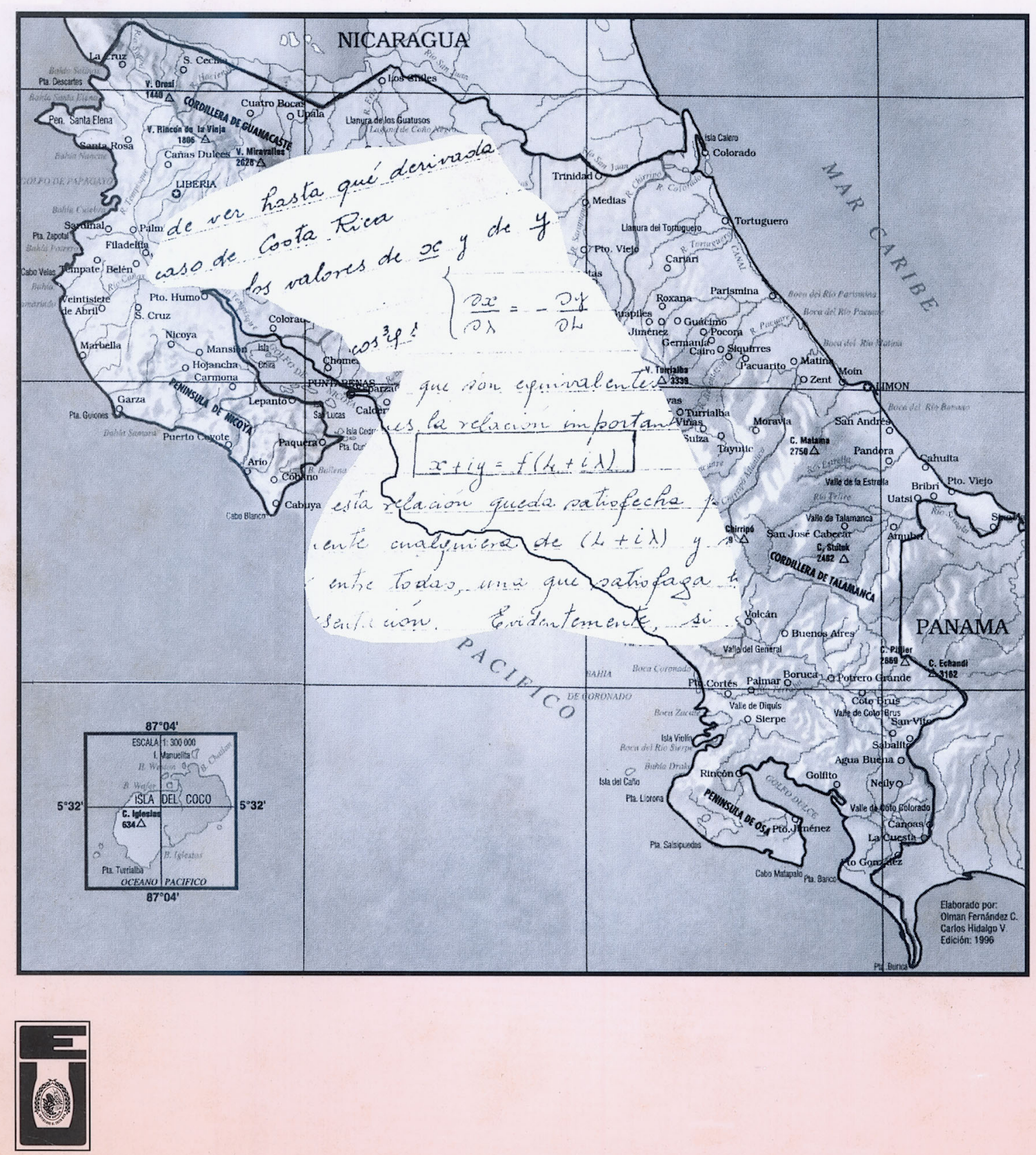


\section{INGENIERIA}

Revista Semestral de la Universidad de Costa Rica Volumen 6, Enero/Junio 1996 Número 1

\section{DIRECTOR}

Rodolfo Herrera J.

\section{CONSEJO EDITORAL}

Víctor Hugo Chacón P. Ismael Mazón G.

Domingo Riggioni C.

\section{CORRESPONDENCIA Y SUSCRIPCIONES}

Editorial de la Universidad de Costa Rica

Apartado Postal 75

2060 Ciudad Universitaria Rodrigo Facio

San José, Costa Rica

\section{CANJES}

Universidad de Costa Rica

Sistema de Bibliotecas, Documentación e Información

Unidad de Selección y Aquisiciones-CANJE

Ciudad Universitaria Rodrigo Facio

San José, Costa Rica

\section{Suscripción anual:}

Costa Rica: $₫ 1000,00$

Otros países: US \$25,00

\section{Número suelto:}

Costa Rica: $\varnothing 750,00$

Otros países: \$ 15,00

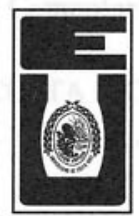


Edición aprobada por la Comisión Editorial de la Universidad de Costa Rica (C) 1998 EDITORIAL DE LA UNIVERSIDAD DE COSTA RICA

Todos los derechos reservados conforme a la ley

Ciudad Universitaria Rodrigo Facio

San José, Costa Rica.

Revisión Filológica: Lorena Rodríguez

Diseño Gráfico, Diagramación y Control de Calidad:

Unidad de Diseño Gráfico de Revistas

Oficina de Publicaciones

Impreso en la Oficina de Publicaciones

de la Universidad de Costa Rica

Revista

620.005

I-46i Ingeniería / Universidad de Costa Rica.

Vol. I, no. 1 (ene./jun. 1991)— _— San José, C. R. : Editorial de la Universidad de Costa Rica, 1991 - (Oficina de Publicaciones de la Universidad de Costa Rica)

v. : il

Semestral.

1. Ingeniería - Publicaciones periódicas.

CCC/BUCR-250

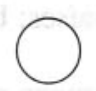




\title{
MODELO MATEMATICO PARA CALCULAR EL FACTOR GEOMETRICO J EN ENGRANES RECTOS
}

\author{
Alejandro Pacheco Molina* \\ Alonso Rodríguez Chacón**
}

\begin{abstract}
Resumen
En este artículo se formula un modelo matemático que permite calcular, en forma analìtica, el factor geométrico J en un engrane recto. El modelo está constituido por una serie de algoritmos concatenados de fácil programación en una computadora. Se generaron curvas del factor $\mathrm{J}$ para diversas condiciones de engranes y se compararon con los valores de diseño referidos por la AGMA, lográndose una excelente correlación.
\end{abstract}

Summary

In this paper, a mathematical model for calculating the geometrical factor $\mathrm{J}$ of spur gears is formulated. The model includes a series of related algorithms which are easily programmed in a computer. The model was tested by generating curves of the $\mathrm{J}$ factor for several gear conditions and plotting them against the AGMA reference values. It was found that the model curves match the AGMA curves very closely.

\section{INTRODUCCION}

El factor geométrico J es un parámetro fundamental para el diseño de engranes en general. El método convencional para obtener el factor $\mathrm{J}$ consiste en consultar los gráficos publicados por la American Gear Manufacturers Association (AGMA), en los cuales se expresa dicho factor como una función de los números de dientes de los engranes conectados. Este método tradicional, además de lento, está sujeto a las imprecisiones propias de una lectura gráfica; aún más, el hecho más serio es que impide agilizar el cálculo de engranes por medio de un programa de computadora.

Desafortunadamente, AGMA no publica el modelo matemático que se emplea para desarrollar sus gráficas y tablas del factor J. Por esa razón, y con miras a desarrollar un programa de computadora para el diseño de engranes rectos, es que se elaboró como primer paso el modelo matemático que permite calcular el factor J que se presenta en este artículo.

\section{PARAMETROS UTILIZADOS EN LOS MODELOS MATEMATICOS}

En la figura No. 1 se muestran los parámetros geométricos principales que intervienen en la formulación del modelo matemático. Otros parámetros de importancia que no se muestran en la figura, se definen en la siguiente lista:

Ingeniero Mecánico M.Sc. Profesor de la Escuela de Ingeniería Mecánica, Universidad de Costa Rica.

** Ingeniero Mecánico 
$\mathrm{a}=$ adendo

$\mathrm{b}=$ dedendo

$\mathrm{O}=$ centro del engrane a calcular

$\mathrm{O}_{1}=$ centro del engrane compañero $\mathrm{o}$ acoplador

$\mathrm{P}=$ punto de tangencia entre los círculos de paso

$\mathrm{C}=$ punto de tangencia entre el círculo base de $\mathrm{O} 1$ y la línea de presión

$\mathrm{K}=$ intersección entre el círculo de adendo y la línea de presión; primer punto de contacto

$\mathrm{L}=$ intersección entre el círculo de adendo de $\mathrm{O} 1$ y la línea de presión; último punto de contacto

$\mathrm{E}=$ punto de tangencia entre el círculo base y la línea de presión

$\mathrm{A}=$ centro del círculo de entalle

$\mathrm{R}=$ punto de tangencia entre el círculo de entalle y el perfil de involuta

$\mathrm{H}=$ punto más alto de contacto

$\mathrm{r}_{\mathrm{a}}=$ radio de adendo

$\mathrm{r}_{\mathrm{b}}=$ radio base

$r_{p}=$ radio de paso

$\mathrm{r}_{\mathrm{d}}=$ radio de dedendo

$r_{\mathrm{f}}=$ radio de entalle

$r_{A}=$ distancia entre el centro del engrane $\mathrm{O}$ y el centro del círculo de entalle $\mathrm{A}$

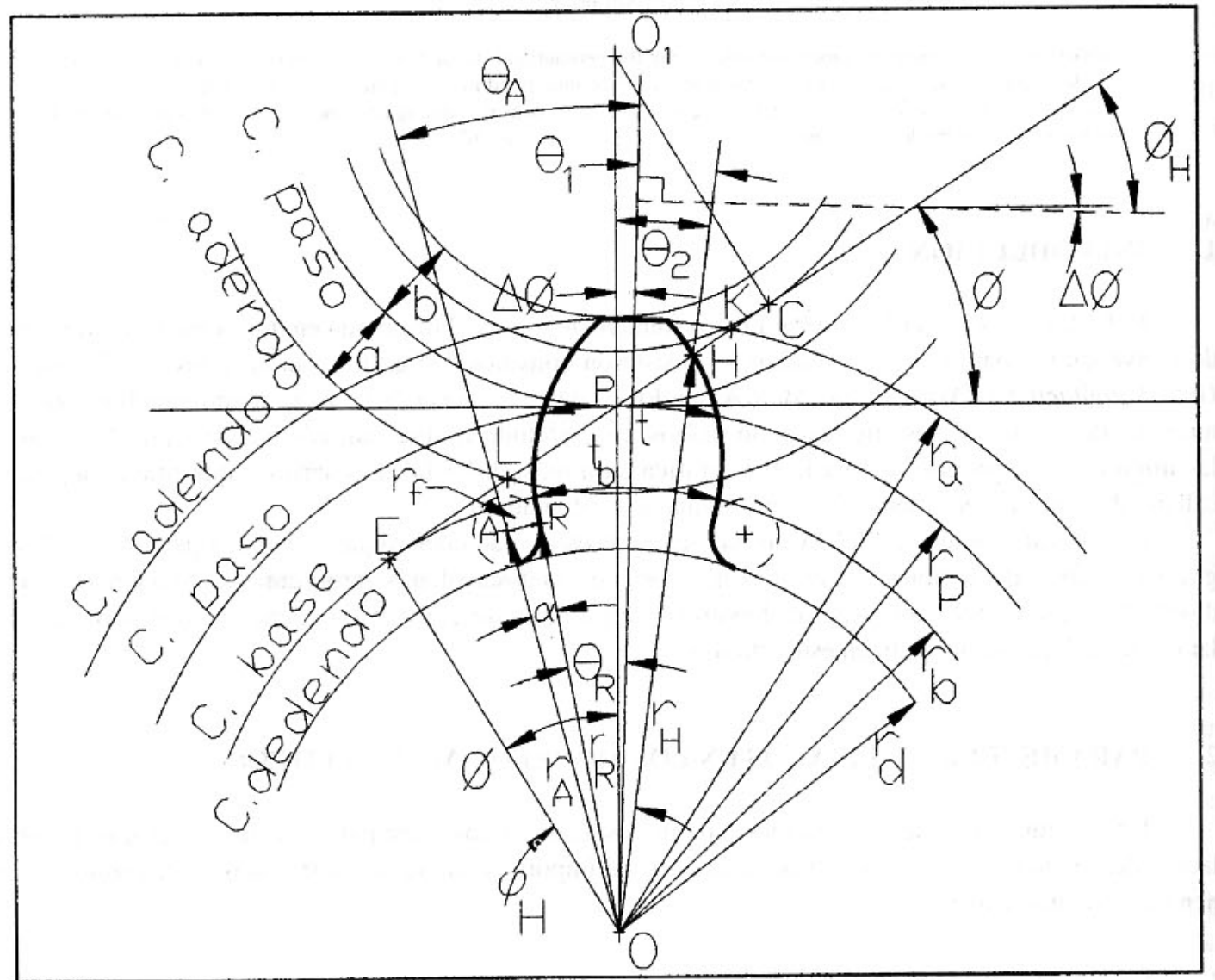

Fig. 1: Nomenclatura de los parámetros utilizados. 
$r_{R}=$ distancia entre el centro del engrane $\mathrm{O}$ y el punto $\mathrm{R}$

$\mathrm{r}_{\mathrm{H}}=$ distancia entre el centro del engrane $\mathrm{O}$ y el punto $\mathrm{H}$

$\mathrm{t}=$ grueso circular del diente

$t_{b}=$ grueso circular del diente en el cl̀rculo base

$\phi=$ ángulo de presión

$\Delta \phi=$ ángulo entre la línea de centro $\mathrm{OO}_{1}$ y la línea central del diente

$\phi_{\mathrm{H}}=$ suma del ángulo de presión $\phi$ y el ángulo $\Delta \phi$

$\theta_{1}=$ ángulo entre la línea $\mathrm{OH}$ y la línea central del diente

$\theta_{2}=$ ángulo entre la línea $\mathrm{OH}$ y la línea de centro $\mathrm{OO}_{1}$

$\theta_{\mathrm{A}}=$ ángulo entre la línea central del diente y la línea $\mathrm{OA}$

$\theta_{\mathrm{R}}=$ ángulo entre la línea central del diente y la línea $\mathrm{OR}$

$\alpha=$ ángulo entre $\mathrm{OA}$ y $\mathrm{OR}$

$\varphi_{\mathrm{H}}=$ ángulo entre $\mathrm{OE}$ y $\mathrm{OH}$

Además se usarán los siguientes parámetros:

$p=$ paso circular

$\mathrm{m}=$ módulo

$\mathrm{N}=$ número de dientes del engrane

$\mathrm{F}=$ ancho de cara del diente

\section{FACTOR GEOMETRICO J PARA ENGRANES RECTOS}

\subsection{Determinación de las ecuaciones analíticas para el cálculo del grosor del diente}

El grosor " $t$ " de un diente de engrane, a una distancia radial " $r$ " a partir del centro del engrane "O" (figura No.2), se puede determinar con la siguiente ecuación (Shigley y Uicker, 1983, p.283):

$$
t=2 r\left(\frac{t_{p}}{2 r_{p}}+i n v \phi-i n v \varphi\right)
$$

donde:

$l_{\mathrm{p}}=\mathrm{p} / 2=\pi / 2 P=\mathrm{m} \pi 2$

$\mathrm{r}_{\mathrm{p}}=\mathrm{d}_{\mathrm{p}} / 2=\mathrm{N} / 2 P=\mathrm{mN} / 2$

$\phi=$ ángulo de presión normal

inv(i) $=\tan (\mathrm{i})-\mathrm{i}, \mathrm{y}$ se conoce como función de involuta del ángulo i (en radianes).

\subsubsection{Grosor del diente en el círculo base}

De la figura No. 2 se puede observar que para un espesor en el círculo base ( $\left.\mathrm{t}_{\mathrm{b}}\right)$, el ángulo $\phi$ es igual a cero.

Además, se sabe que : $r_{b}=r_{p} \cos \phi$

Entonces, de la ecuación (1) :

$$
t_{b}=2 r_{b}\left(\frac{t_{p}}{2 r_{p}}+i n v \phi\right)
$$




\subsubsection{Grosor del diente en el círculo de adendo}

Se tiene que: $r_{a}=r_{p}+a$

De la figura No.2 se deduce que $r_{a}=r_{b} / \cos \varphi_{a}$

$$
\varphi_{a}=\cos ^{-1}\left(\mathrm{r}_{\mathrm{b}} / \mathrm{r}_{\mathrm{b}}\right)
$$

Finalmente de la ecuación (1):

$$
t_{a}=2 r_{a}\left(\frac{t_{p}}{2 r_{p}}+i n v \phi-i n v \varphi_{a}\right)
$$

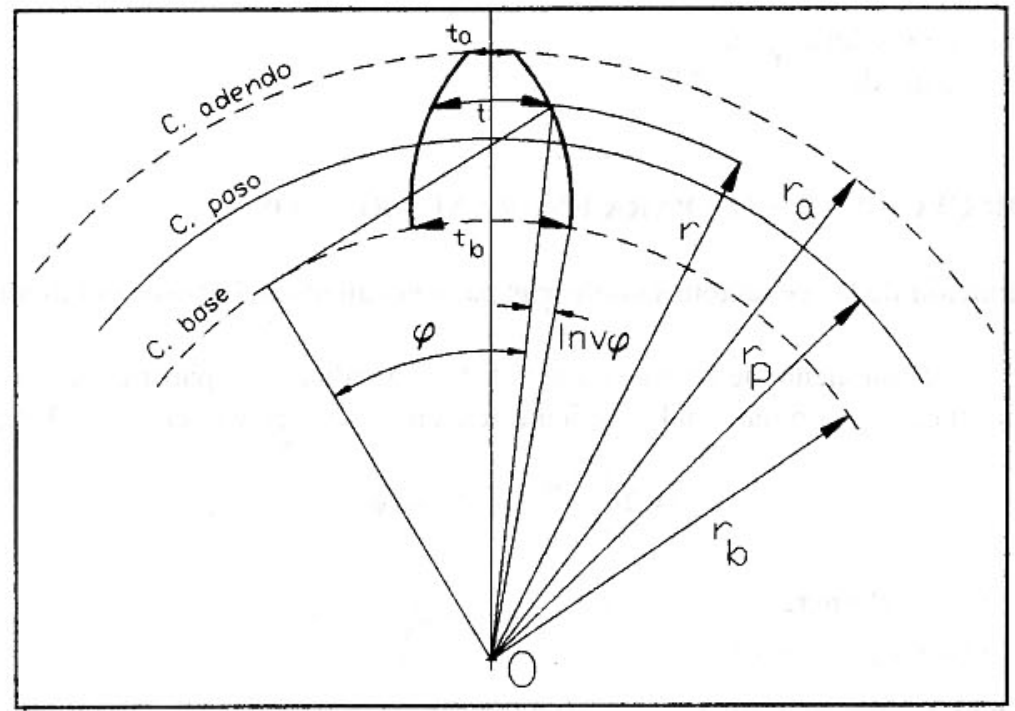

Fig. 2: Relaciones de involumetría.

\subsection{Determinación del punto más alto de contacto}

El punto más alto de contacto " $\mathrm{H}$ " se encuentra a una distancia igual al paso base pb desde el último punto de contacto " $L$ ", como se observa en la figura No. 3. El punto " $\mathrm{H}_{1}$ " es el punto más alto de contacto del engrane compañero. 


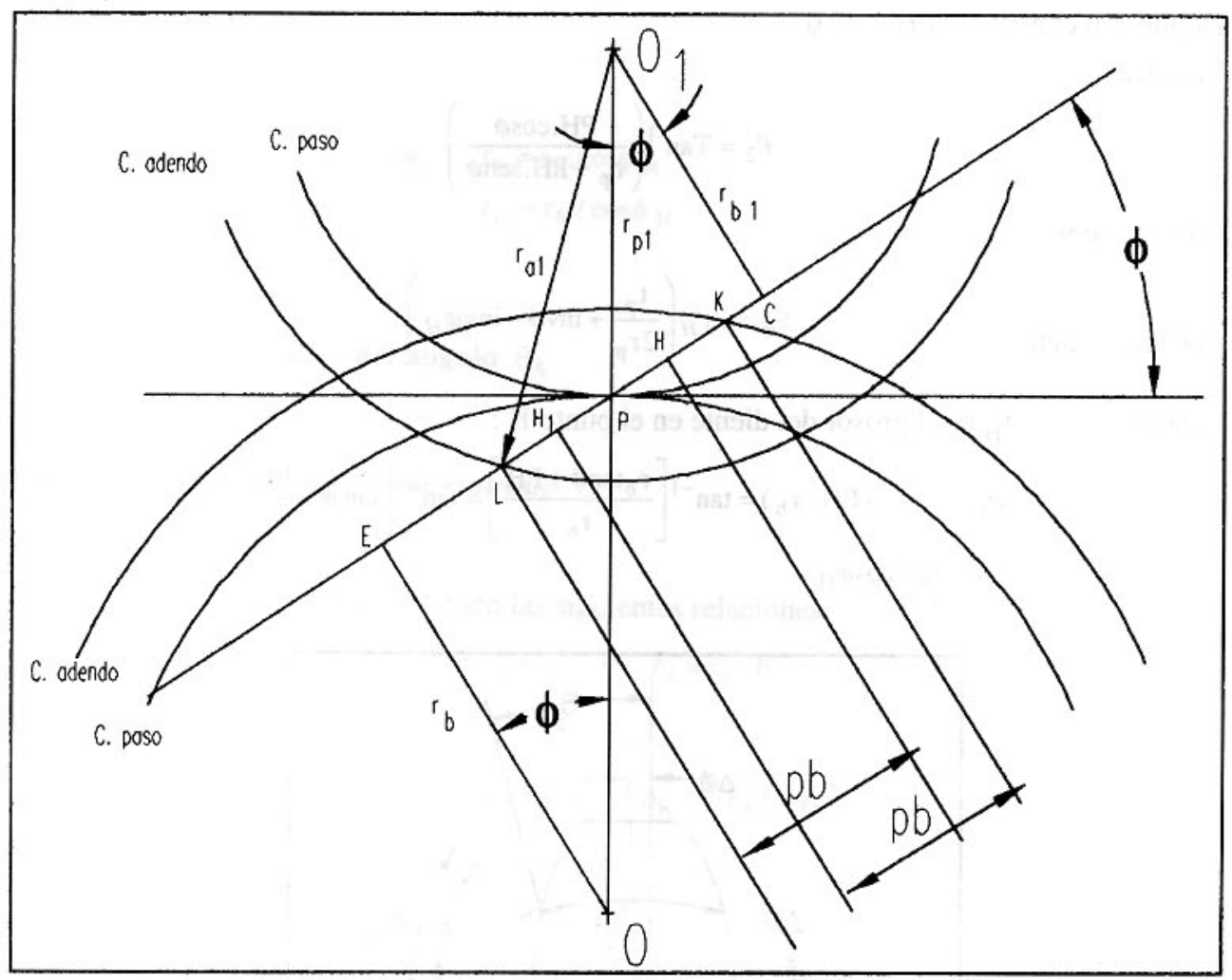

Fig. 3 : Determinación del punto más alto de contacto $H$.

\subsubsection{Determinación de $\mathrm{PH}$}

De la figura No. 3, se tiene que :

$$
\begin{aligned}
& \mathrm{p}_{\mathrm{b}}=\mathrm{p} \cdot \cos \phi=\pi \cos \phi / \mathrm{P} \\
& \mathrm{PL}=\mathrm{CL}-\mathrm{PC}=\sqrt{\mathrm{r}_{\mathrm{a} 1}^{2}-\mathrm{r}_{\mathrm{bl}}^{2}}-\mathrm{r}_{\mathrm{pl}} \operatorname{sen} \phi \\
& \operatorname{con} \mathrm{r}_{\mathrm{a} 1}=\mathrm{r}_{\mathrm{pl}}+\mathrm{a} \text { y } \mathrm{r}_{\mathrm{bl}}=\mathrm{r}_{\mathrm{pl}} \cos \phi
\end{aligned}
$$

donde el subíndice "1" indica el radio del engrane compañero.

Entonces: $\mathrm{PH}=\mathrm{LH}$ - PL, pero como $\mathrm{LH}=\mathrm{p}_{\mathrm{b}}$, entonces:

$$
\mathrm{PH}=\mathrm{p}_{\mathrm{b}}-\mathrm{PL}
$$

\subsubsection{Determinación de $\Delta \phi$}

De la figura No. 4 , se sabe que : $\Delta \phi=\theta_{2}-\theta_{1}$

- Cálculo de $\theta_{2}$ :

$$
\operatorname{Tan} \theta_{2}=\left(\frac{\mathrm{PH} \cdot \cos \phi}{\mathrm{OP}+\mathrm{PH} \cdot \operatorname{sen} \phi}\right)=\left(\frac{\mathrm{PH} \cdot \cos \phi}{\mathrm{r}_{\mathrm{p}}+\mathrm{PH} \cdot \operatorname{sen} \phi}\right)
$$


Donde son conocidos $\mathrm{PH}, \mathrm{r}_{\mathrm{p}}$ y $\phi$.

Entonces:

$$
\theta_{2}=\operatorname{Tan}^{-1}\left(\frac{P H \cdot \cos \phi}{r_{p}+P H \cdot \operatorname{sen} \phi}\right)
$$

Cálculo de $\theta_{1}$ :

Por involumetría :

$$
\mathrm{t}_{H}=2 \mathrm{r}_{H}\left(\frac{\mathrm{t}_{\mathrm{p}}}{2 \mathrm{r}_{\mathrm{p}}}+\operatorname{inv} \phi-\operatorname{inv} \varphi_{H}\right),
$$

donde: $\quad t_{H}$ es el grosor del diente en el punto $\mathrm{H}$ :

$$
\begin{aligned}
& \varphi_{\mathrm{H}}=\tan ^{-1}\left(E H / r_{b}\right)=\tan ^{-1}\left[\frac{r_{b} \tan \phi+P H}{r_{b}}\right]=\tan ^{-1}\left[\tan \phi+\frac{P H}{r_{b}}\right] \\
& r_{H}=r_{b} / \cos \varphi_{H} .
\end{aligned}
$$

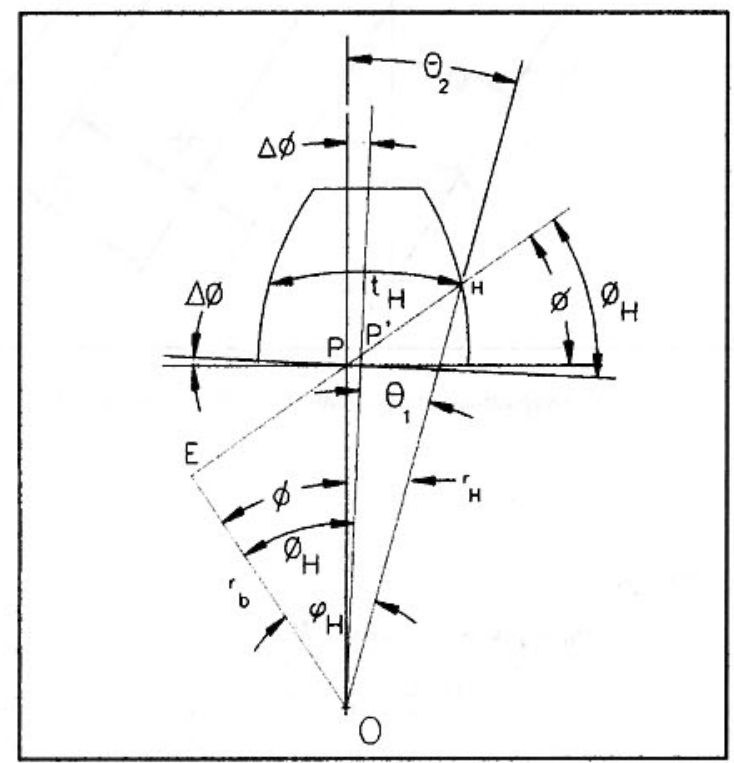

Fig. 4 : Punto más alto de contacto $\mathrm{H}$.

Usando la relación : ángulo * radio $=$ arco, se tiene que

$$
\theta_{1} \cdot \mathrm{r}_{\mathrm{H}}=\mathrm{t}_{\mathrm{H}} / 2 \Rightarrow \theta_{1}=\frac{\mathrm{t}_{\mathrm{H}}}{2 \cdot \mathrm{r}_{\mathrm{H}}}(\mathrm{rad})
$$

Finalmente:

$$
\begin{aligned}
& \Delta \phi=\theta_{2}-\theta_{1}, \mathrm{y} \\
& \phi_{\mathrm{H}}=\phi+\Delta \phi
\end{aligned}
$$




\subsubsection{Determinación del radio $\mathbf{r}_{\mathbf{p}}$,}

De la figura No. 4, se tiene que: $\quad r_{b}=r_{p}, \cos \phi_{H}$

$$
r_{p},=r_{b} / \cos \phi_{H}
$$

\subsection{Determinación analítica del ángulo $\theta_{\mathbf{A}}$}

\subsubsection{Para dientes con perfil completo de evolvente}

A partir de la figura No. 5 se obtienen las siguientes relaciones:

$$
r_{A}=r_{d}+r_{f} \quad \text { y } \quad r_{d}=r_{p}-b
$$

Del triángulo OAF :

$$
\mathrm{AF}=\mathrm{AR}+\mathrm{RF}=\mathrm{r}_{\mathrm{f}}+\rho_{\mathrm{R}}=\sqrt{\mathrm{r}_{\mathrm{A}}^{2}-\mathrm{r}_{\mathrm{b}}^{2}}
$$

donde: $\quad \rho_{\mathrm{R}}=\sqrt{\mathrm{r}_{\mathrm{A}}^{2}-\mathrm{r}_{\mathrm{b}}^{2}}-\mathrm{r}_{\mathrm{f}}$

Además, se tiene que: $\tan \varphi_{\mathrm{R}}=\rho_{\mathrm{R}} / \mathrm{r}_{\mathrm{b}}$, entonces: $\varphi_{\mathrm{R}}=\operatorname{Tan}^{-1}\left(\rho_{\mathrm{R}} / \mathrm{r}_{\mathrm{b}}\right)$

$\mathrm{r}_{\mathrm{R}}=\sqrt{\rho_{\mathrm{R}}^{2}+\mathrm{r}_{\mathrm{b}}^{2}}$

Por involumetría (ecuación 1) se obtiene que:

y

$$
\begin{aligned}
& \frac{\mathrm{t}_{\mathrm{R}}}{2}=\mathrm{r}_{R}\left(\frac{\mathrm{t}_{\mathrm{p}}}{2 \mathrm{r}_{\mathrm{p}}}+\operatorname{inv} \phi-\operatorname{inv} \varphi_{R}\right) \\
& \mathrm{t}_{\mathrm{R}} / 2=\mathrm{r}_{\mathrm{R}} \cdot \theta_{\mathrm{R}}
\end{aligned}
$$

Igualando las ecuaciones anteriores:

$$
\theta_{R}=\frac{\mathrm{t}_{\mathrm{p}}}{2 \mathrm{r}_{\mathrm{p}}}+\operatorname{inv} \phi-\operatorname{inv} \varphi_{R}
$$

Entonces:

$$
\begin{aligned}
& \beta=\varphi_{\mathrm{R}}-\theta_{\mathrm{R}} \\
& \mathrm{AG}=\mathrm{RH}+\mathrm{r}_{\mathrm{f}} \cdot \cos \beta=\mathrm{r}_{\mathrm{R}} \cdot \operatorname{sen} \theta_{\mathrm{R}}+\mathrm{r}_{\mathrm{f}} \cdot \cos \beta
\end{aligned}
$$




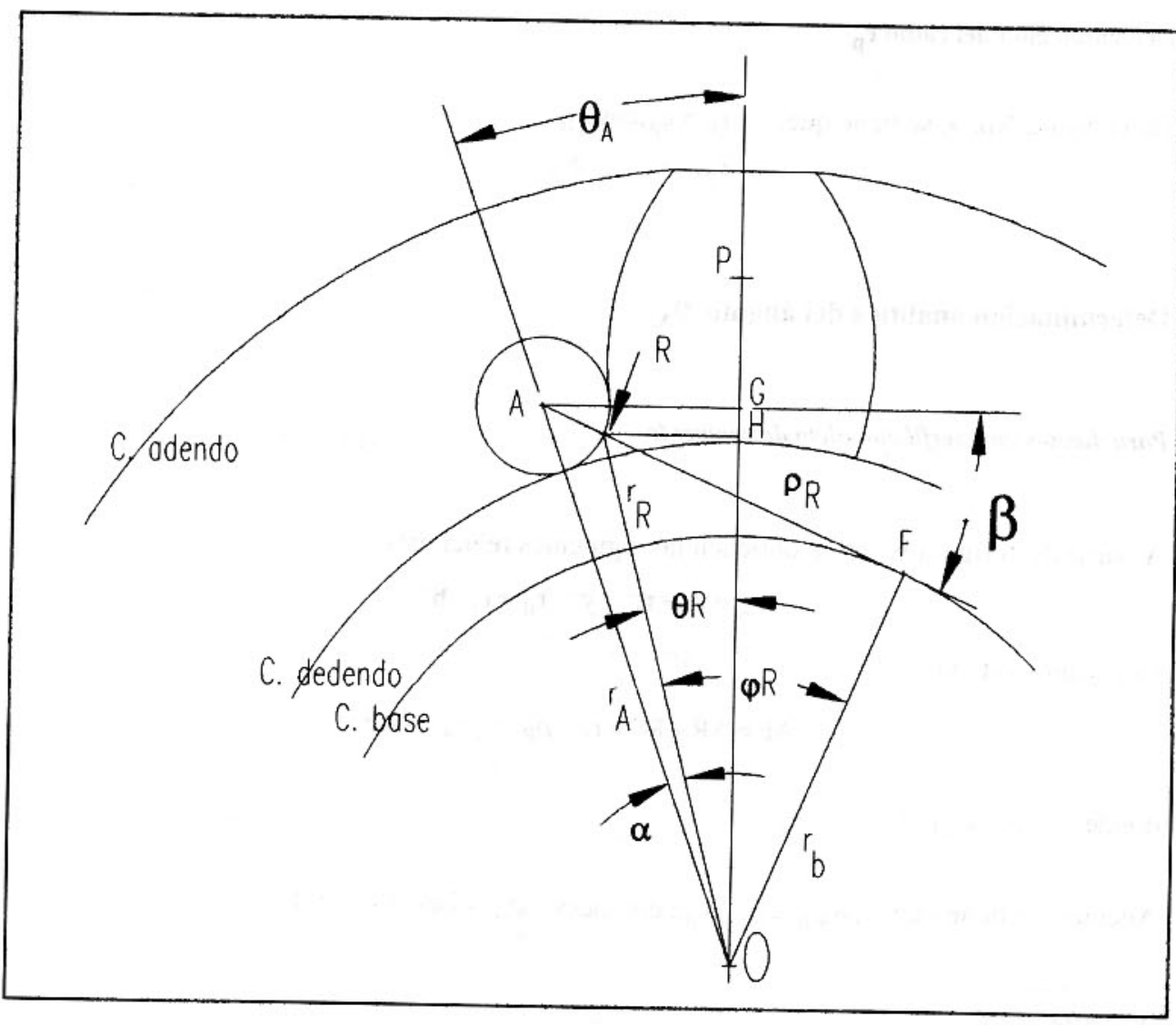

Fig. 5 : Determinación de $\theta_{\mathrm{A}}$ para perfil completo de evolvente.

Finalmente se obtiene que :

$$
\theta_{\mathrm{A}}=\operatorname{sen}^{-1}\left(\frac{\mathrm{AG}}{\mathrm{r}_{\mathrm{A}}}\right)
$$

\subsubsection{Para dientes con entalle por debajo del círculo base}

Refiriéndose a la figura No. 6, se obtienen las siguientes relaciones:

$$
r_{A}=r_{d}+r_{f}
$$

Por la relación ángulo $=$ arco/radio: $\theta_{\mathrm{R}}=\mathrm{t}_{\mathrm{b}} / 2 \mathrm{r}_{\mathrm{b}}$ y $t_{b}$ se conoce de la ecuación (2).

Del triángulo OAR:

$$
\mathrm{r}_{\mathrm{A}} \operatorname{sen} \alpha=\mathrm{r}_{\mathrm{f}} \quad \Rightarrow \quad \alpha=\operatorname{sen}^{-1}\left(\mathrm{r}_{\mathrm{f}} / \mathrm{r}_{\mathrm{A}}\right)
$$


Finalmente:

$$
\theta_{\mathrm{A}}=\theta_{\mathrm{R}}+\alpha=\frac{\mathrm{t}_{\mathrm{b}}}{2 \mathrm{r}_{\mathrm{b}}}+\operatorname{sen}^{-1}\left(\frac{\mathrm{r}_{\mathrm{f}}}{\mathrm{r}_{\mathrm{A}}}\right)
$$

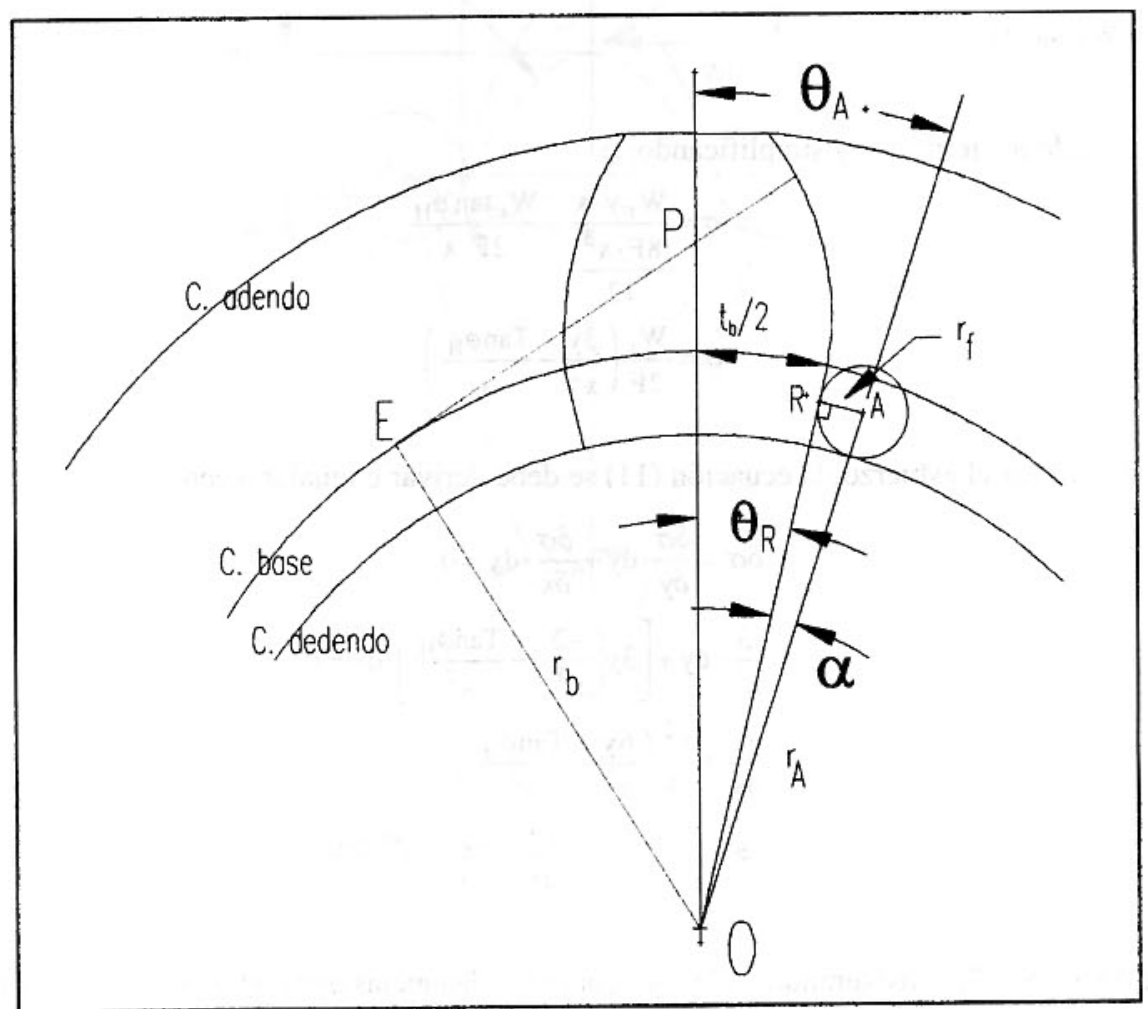

Fig. 6: Determinación de (A para dientes con entalle por debajo del círculo base.

\subsection{Determinación de la sección crítica al esfuerzo de flexión en un diente de engrane recto}

La sección crítica en un diente de engrane recto se encuentra en la sección que pasa por el punto $\mathrm{S}$ de la figura No. 7. Para ubicar el punto S, se suele utilizar el criterio gráfico convencional de la parábola: la sección crítica se encuentra en el punto de tangencia entre el círculo de entalle, con centro en $\mathrm{A}$, y una función parabólica desarrollada a partir del punto P'.

$\mathrm{Si}$ se toma en cuenta la componente radial $(\mathrm{Wr})$ de la fuerza $\mathrm{W}$ que se aplica al diente del engrane (figura No. 7), se tiene la siguiente relación para el esfuerzo resultante en cualquier sección genérica a una distancia "y" por debajo de P'.

$$
\sigma=\frac{\mathrm{Mc}}{\mathrm{I}}-\frac{\mathrm{W}_{\mathrm{r}}}{\mathrm{A}} \quad \text {, donde: }
$$




$$
\begin{aligned}
& M=M_{t} \cdot y \\
& c=x \\
& I=\left[(2 x)^{3} \cdot F\right] / 12 \\
& A=F \cdot(2 x) \\
& W_{r}=W_{t} \cdot \tan \phi_{H}
\end{aligned}
$$

Sustituyendo los términos y simplificando:

$$
\begin{gathered}
\sigma=\frac{\mathrm{W}_{\mathrm{t} \cdot \mathrm{y} \cdot \mathrm{x}}}{\frac{8 \mathrm{~F} \cdot \mathrm{x}^{3}}{12}}-\frac{\mathrm{W}_{\mathrm{t}} \tan \phi_{\mathrm{H}}}{2 \mathrm{~F} \cdot \mathrm{x}} \\
\sigma=\frac{\mathrm{W}_{\mathrm{t}}}{2 \mathrm{~F}}\left(\frac{3 \mathrm{y}}{\mathrm{x}^{2}}-\frac{\operatorname{Tan} \phi_{\mathrm{H}}}{\mathrm{x}}\right)
\end{gathered}
$$

Para maximizar el esfuerzo, la ecuación (11) se debe derivar e igualar a cero:

$$
\begin{aligned}
& \delta \sigma=\frac{\delta \sigma}{\delta \mathrm{y}} \cdot \mathrm{dy}+\frac{\delta \sigma}{\delta \mathrm{x}} \cdot \mathrm{dx}=0 \\
& \frac{3}{\mathrm{x}^{2}} \cdot \mathrm{dy}+\left[3 y\left(\frac{-2}{\mathrm{x}^{3}}\right)+\frac{\operatorname{Tan} \phi_{\mathrm{H}}}{\mathrm{x}^{2}}\right] \cdot \mathrm{dx}=0 \\
& \frac{\mathrm{dy}}{\mathrm{dx}}=\frac{\mathrm{x}^{2}}{3}\left(\frac{6 \mathrm{y}}{\mathrm{x}^{3}}-\frac{\operatorname{Tan} \phi_{\mathrm{H}}}{\mathrm{x}^{2}}\right) \\
& \Rightarrow \quad \frac{\mathrm{dy}}{\mathrm{dx}}=2 \frac{\mathrm{y}}{\mathrm{x}}-\frac{\operatorname{Tan} \phi_{\mathrm{H}}}{3}
\end{aligned}
$$

De la figura No. 7, considerando " $\mathrm{x}$ " $\mathrm{y}$ " $y$ " como las distancias entre Pí y el punto S, se puede obtener las siguientes relaciones:

$$
\begin{aligned}
& y=O P^{\prime}-O G+G I=r_{p^{\prime}}-r_{A} \cdot \cos \theta_{A}+r_{f} \cdot \operatorname{sen} \beta s \\
& x=r_{A} \cdot \operatorname{sen} \theta_{A}-r_{f} \cdot \cos \beta s
\end{aligned}
$$

Utilizando la siguiente relación:

$$
\frac{\mathrm{dy}}{\mathrm{dx}}=\frac{\delta \mathrm{y} / \delta \beta_{\mathrm{s}}}{\delta \mathrm{x} / \delta \beta_{\mathrm{s}}}=\frac{\mathrm{r}_{\mathrm{f}} \cdot \cos \beta_{\mathrm{s}}}{-\mathrm{r}_{\mathrm{f}} \cdot\left(-\operatorname{sen} \beta_{\mathrm{s}}\right)}=\cot \beta_{\mathrm{s}}
$$

Finalmente, sustituyendo en la ecuación (12):

$$
\operatorname{Cot} \beta_{\mathrm{s}}=\frac{2\left(\mathrm{r}_{\mathrm{p}^{\prime}}-\mathrm{r}_{\mathrm{A}} \cdot \cos \theta_{\mathrm{A}}+\mathrm{r}_{\mathrm{f}} \cdot \operatorname{sen} \beta_{\mathrm{s}}\right)}{\mathrm{r}_{\mathrm{A}} \cdot \operatorname{sen} \theta_{\mathrm{A}}-\mathrm{r}_{\mathrm{f}} \cdot \cos \beta_{\mathrm{s}}}-\frac{\operatorname{Tan} \phi_{\mathrm{H}}}{3}
$$




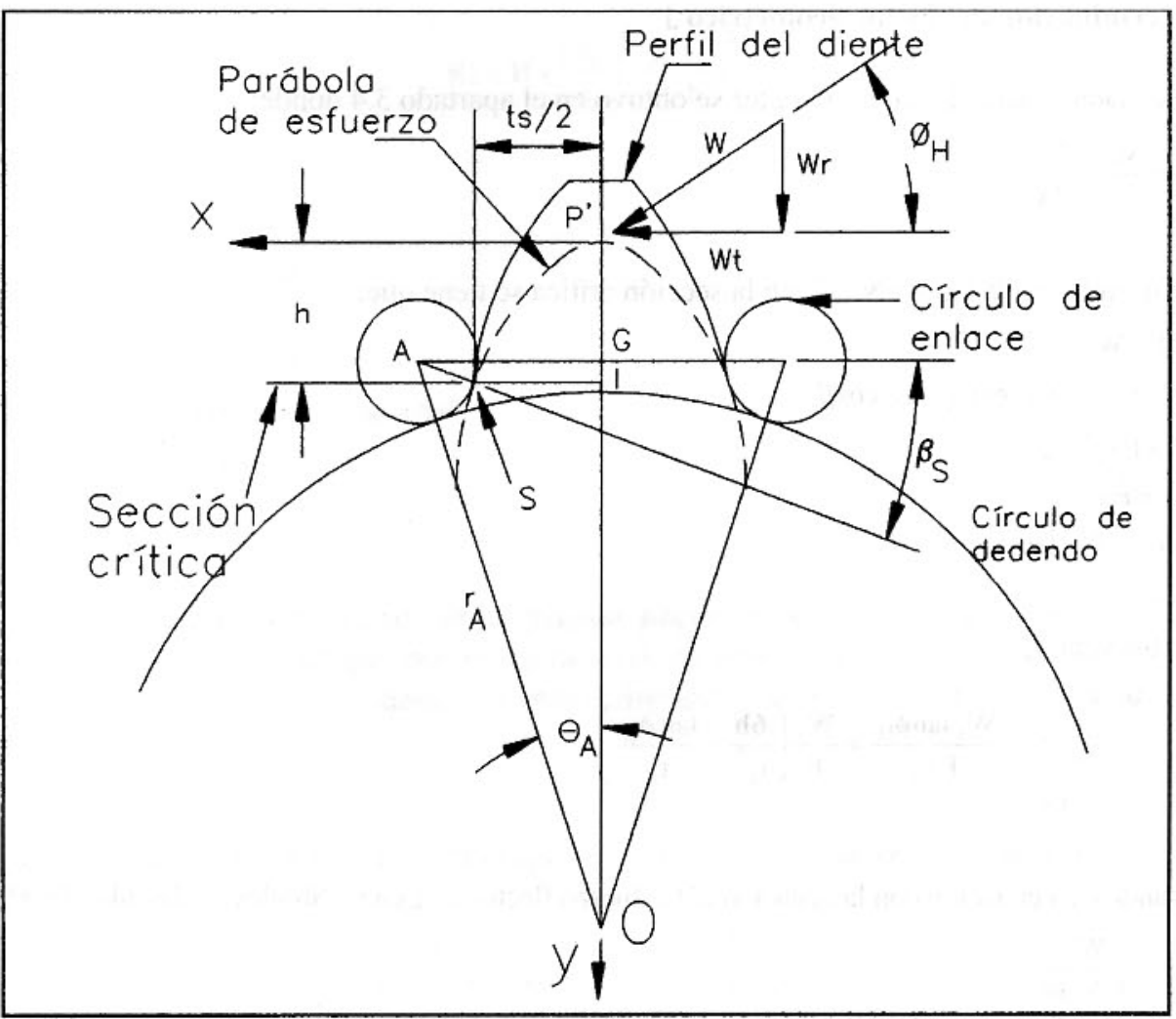

Fig. 7: Determinación de la sección crítica.

El punto "S" obtenido por medio de esta técnica es más preciso que el obtenido por la técnica de la parábola, en vista de que esta última desprecia la influencia de la carga radial.

La ecuación (13) se puede resolver utilizando un método numérico iterativo para determinar la solución de ecuaciones de una variable; se recomienda el método de la secante (Burden y Faires, 1985, p. 59).

\subsection{Determinación del brazo de palanca entre la carga $W$ y la sección crítica}

El brazo de palanca se define como la distancia " $h$ " entre el punto P' y la sección crítica (figura No. 7). Entonces:

$$
\begin{aligned}
& \mathrm{h}=\mathrm{OP}^{\prime}-\mathrm{OI}=\mathrm{r}_{\mathrm{p}^{\prime}}-\mathrm{r}_{\mathrm{I}} \\
& \mathrm{r}_{\mathrm{I}}=\mathrm{OG}-\mathrm{GI} \\
& \mathrm{OG}=\mathrm{r}_{\mathrm{A}} \cdot \cos \theta_{\mathrm{A}} \\
& \mathrm{GI}=\mathrm{r}_{\mathrm{f}} \cdot \operatorname{sen} \beta_{\mathrm{s}} \quad \Rightarrow \quad \mathrm{r}_{\mathrm{I}}=\mathrm{r}_{\mathrm{A}} \cdot \cos \theta_{\mathrm{A}}-\mathrm{r}_{\mathrm{f}} \cdot \operatorname{sen} \beta_{\mathrm{s}}
\end{aligned}
$$

Finalmente:

$$
\mathrm{h}=\frac{\mathrm{r}_{\mathrm{b}}}{\cos \phi_{\mathrm{H}}}-\left(\mathrm{r}_{\mathrm{A}} \cdot \cos \theta_{\mathrm{A}}-\mathrm{r}_{\mathrm{f}} \cdot \operatorname{sen} \beta_{\mathrm{s}}\right)
$$




\subsection{Determinación del factor geométrico J}

La ecuación para el esfuerzo flector se obtuvo en el apartado 3.4 donde:

$$
\sigma=\frac{\mathrm{Mc}}{\mathrm{I}}-\frac{\mathrm{W}_{\mathrm{r}}}{\mathrm{A}}
$$

Refiriéndose a la figura No. 7, en la sección crítica se tiene que:

$$
\begin{aligned}
& \mathrm{M}=\mathrm{W}_{\mathrm{t}} \mathrm{h} \\
& \mathrm{c}=\mathrm{t}_{\mathrm{s}} / 2=\mathrm{r}_{\mathrm{A}} \cdot \operatorname{sen} \theta_{\mathrm{A}}-\mathrm{r}_{\mathrm{f}} \cdot \cos \beta_{\mathrm{s}} \\
& \mathrm{I}=\mathrm{F} \cdot \mathrm{t}_{\mathrm{s}}{ }^{3} / 12 \\
& A=\mathrm{F}_{\mathrm{t}} \\
& \mathrm{W}_{\mathrm{r}}=\mathrm{W}_{\mathrm{t}} \cdot \tan \phi_{\mathrm{H}}
\end{aligned}
$$

Sustituyendo,

$$
\sigma=\frac{\mathrm{W}_{\mathrm{t}}, \mathrm{h}^{\mathrm{t}_{\mathrm{s}}} / 2}{\mathrm{~F}_{\mathrm{t}} \mathrm{t}_{\mathrm{s}}^{3} / 12}-\frac{\mathrm{W}_{\mathrm{t}} \cdot \tan \phi_{\mathrm{H}}}{\mathrm{F} \cdot \mathrm{t}_{\mathrm{s}}}=\frac{\mathrm{W}_{\mathrm{t}}}{\mathrm{F}}\left(\frac{6 \mathrm{~h}}{\mathrm{t}_{\mathrm{s}}{ }^{2}}-\frac{\tan \phi_{\mathrm{H}}}{\mathrm{t}_{\mathrm{s}}}\right),
$$

y comparando ésta ecuación con la ecuación del esfuerzo flector de Lewis (Shigley y Mischke, 1990, p. 662):

$$
\sigma=\frac{W_{t}}{\text { F.Y.m }}
$$

Se obtiene, por analogía, un factor geométrico Y, diferente al de Lewis, e igual a:

$$
\mathrm{Y}=\frac{1}{\left(\frac{6 \mathrm{~h}}{\mathrm{t}_{\mathrm{s}}{ }^{2}}-\frac{\tan \phi_{\mathrm{H}}}{\mathrm{ts}}\right) \cdot \mathrm{m}} \text {, donde } \mathrm{m} \text { es el módulo. }
$$

Finalmente, para obtener el factor geométrico J como lo define AGMA, se debe tomar en cuenta la concentración de esfuerzos $\mathrm{K}_{\mathrm{f}}$ y la compartición de carga mN (Shigley y Mischke, 1990, p. 678):

$$
J=\frac{Y}{K_{f} \cdot m_{N}}
$$

Para los engranes rectos, el factor de compartición de carga $\mathrm{m}_{\mathrm{N}}$ es igual a la unidad.

El factor de concentración de esfuerzos $\mathrm{K}_{\mathrm{f}}$ se puede determinar con la siguiente ecuación:

$$
\mathrm{K}_{\mathrm{f}}=\left(\mathrm{K}_{\mathrm{t}}-1\right) \cdot \mathrm{q}+1
$$

donde $\mathrm{q}$ es la sensibilidad de la muesca que conservadoramente se considerará igual a la unidad, y $\mathrm{K}_{\mathrm{t}}$ es el factor geométrico de concentración de esfuerzo en el entalle del diente. El valor de $\mathrm{K}_{\mathrm{t}}$, para engranes fabricados por conformación, se puede obtener de la relación (Shigley y Mitchell, 1985, p. 635): 


$$
\mathrm{K}_{\mathrm{t}}=\mathrm{H}+\left(\frac{\mathrm{t}_{\mathrm{s}}}{\mathrm{r}_{\mathrm{s}}}\right)^{\mathrm{L}} *\left(\frac{\mathrm{t}_{\mathrm{s}}}{\mathrm{h}}\right)^{\mathrm{M}}
$$

donde:

$$
\begin{aligned}
\mathrm{H} & =0.340-0.4583662 . \phi \\
\mathrm{L} & =0.316-04583662 . \phi \\
\mathrm{M} & =0.290+0.4583662 . \phi \\
\phi & =\text { ángulo de presión nominal (rad) } \\
\mathrm{t}_{\mathrm{s}} & =2 \mathrm{r}_{\mathrm{A}} \operatorname{sen} \theta_{\mathrm{A}}-2 \mathrm{r}_{\mathrm{f}} \cos \beta_{\mathrm{s}}
\end{aligned}
$$

El modelo matemático presentado en las páginas anteriores permite el cálculo secuencial de los numerosos parámetros intermedios que definen al factor $\mathrm{J}$, de acuerdo a la ecuación (16). Para ello se requieren como datos los números de dientes de ambos engranes y el sistema de dientes normalizado al que pertenecen.

\section{RESULTADOS DEL FACTOR GEOMETRICO J PARA ENGRANES RECTOS}

Las figuras No. 8 y No. 9 muestran gráficos del valor de J para diferentes números de dientes del engrane. Los gráficos se realizan para valores dados de número de dientes del engrane compañero o acoplador " $\mathrm{N}_{\mathrm{c}}$ ", del ángulo de presión normal " $\phi_{\mathrm{n}}$ " y para sistemas de dientes de altura completa : $\mathrm{a}=\mathrm{m}, \mathrm{b}=$ $1,25 \mathrm{~m}$.

La primera observación que se puede obtener a partir de los gráficos, es el comportamiento similar que tienen las curvas obtenidas y las curvas de AGMA. El valor de $\mathbf{J}$ crece rápidamente en un primer segmento de la curva, para luego mantener un crecimiento mínimo hasta el final.

En cualquiera de los casos graficados, los resultados obtenidos de $\mathbf{J}$ para valores de número de dientes menores de aproximadamente 100 , son ligeramente menores que los datos dados por la AGMA. La diferencia entre los resultados disminuye conforme el número de dientes crece. Sin embargo, los valores obtenidos de $\mathbf{J}$ menores representan resultados relativamente más conservadores, que los de la AGMA, de manera que el diseño se realiza con seguridad. En otras palabras, el valor obtenido J menor que el de AGMA produce resultados de esfuerzos por flexión mayores, y por lo tanto más conservadores.

Para los casos de ángulo de presión de $25^{\circ}$, figuras No. 10 y No. 11,10 resultados obtenidos y los de AGMA se mantienen prácticamente iguales a partir de un número de dientes superior a 100.

Resultados para ángulos de presión diferentes de $20^{\circ}$ o $25^{\circ}$ no se muestran debido a que ángulos diferentes de los anteriores no son utilizados normalmente. 


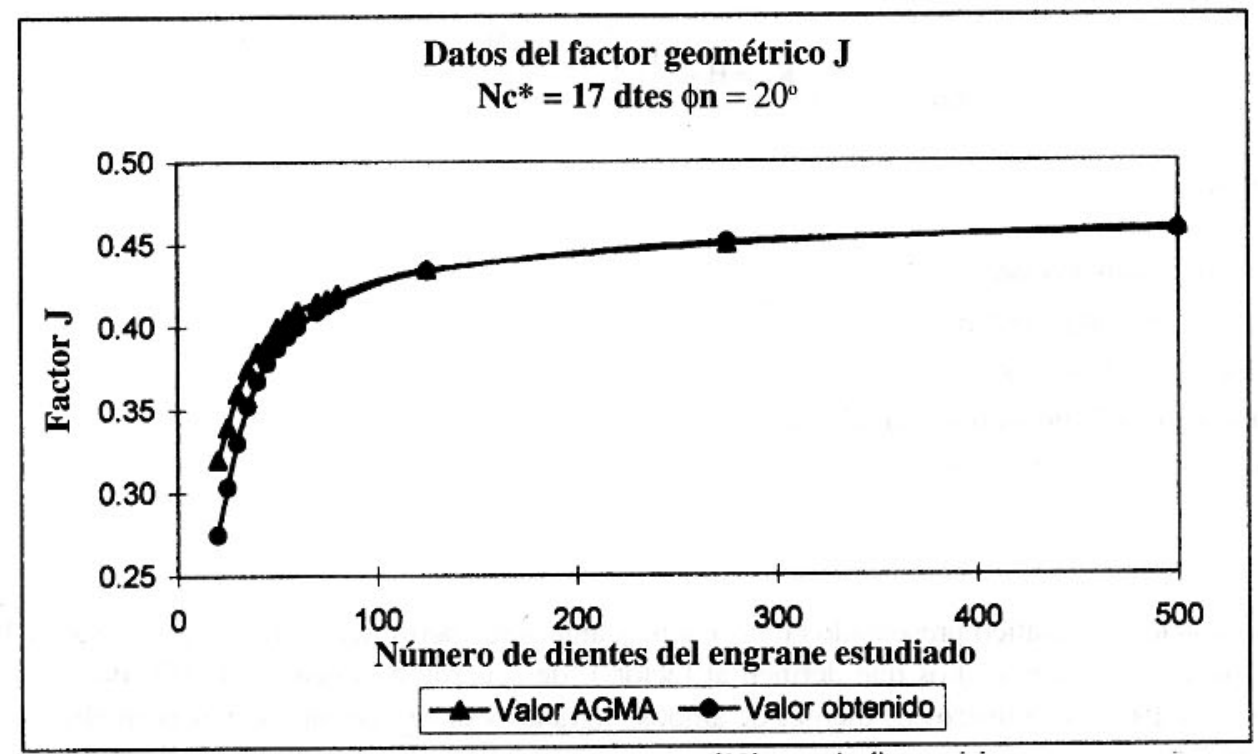

*Número de dientes del engrane compañero

Fig. 8: Comparación del factor J para engranes rectos.

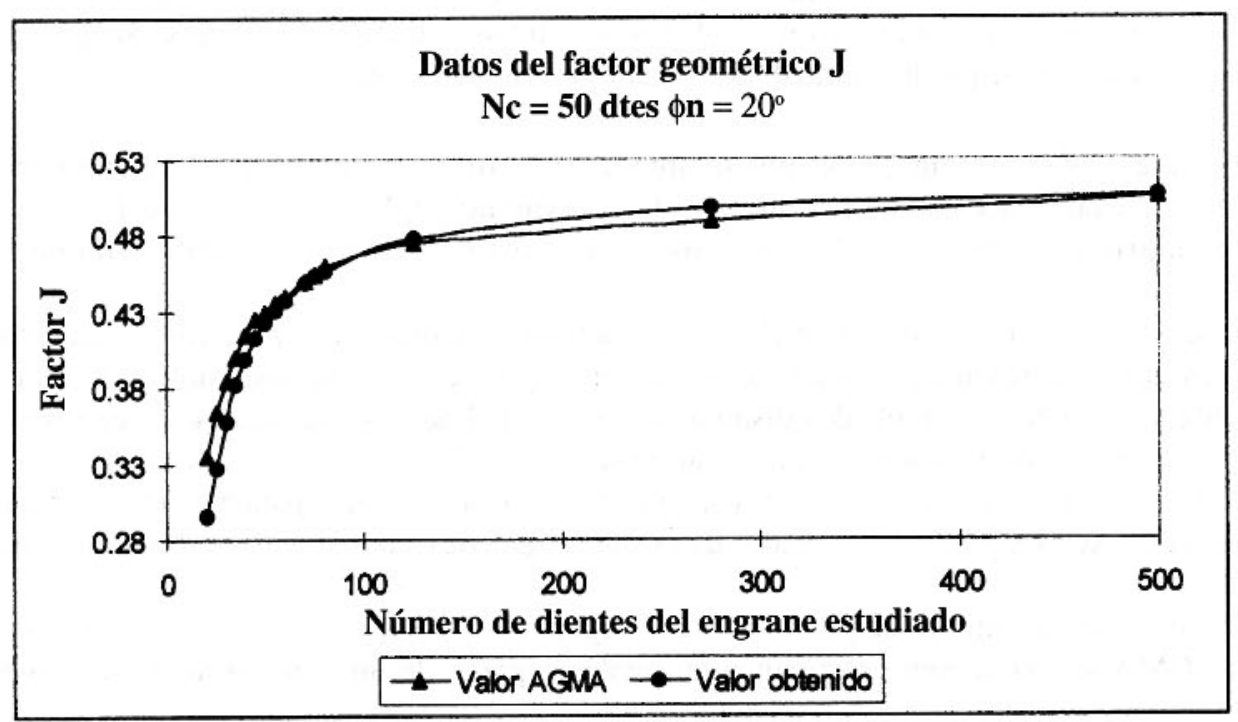

Fig. 9: Comparación del factor J para engranes rectos. 


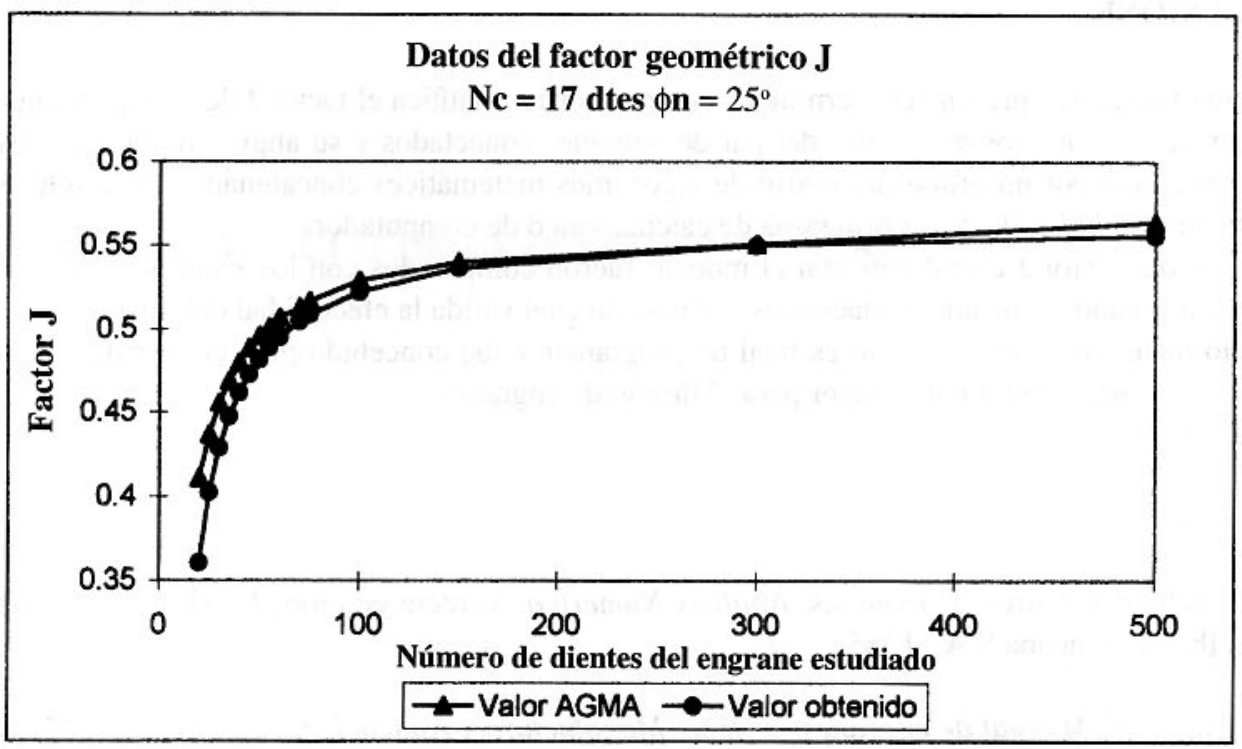

Fig. 10: Comparación del factor J para engranes rectos.

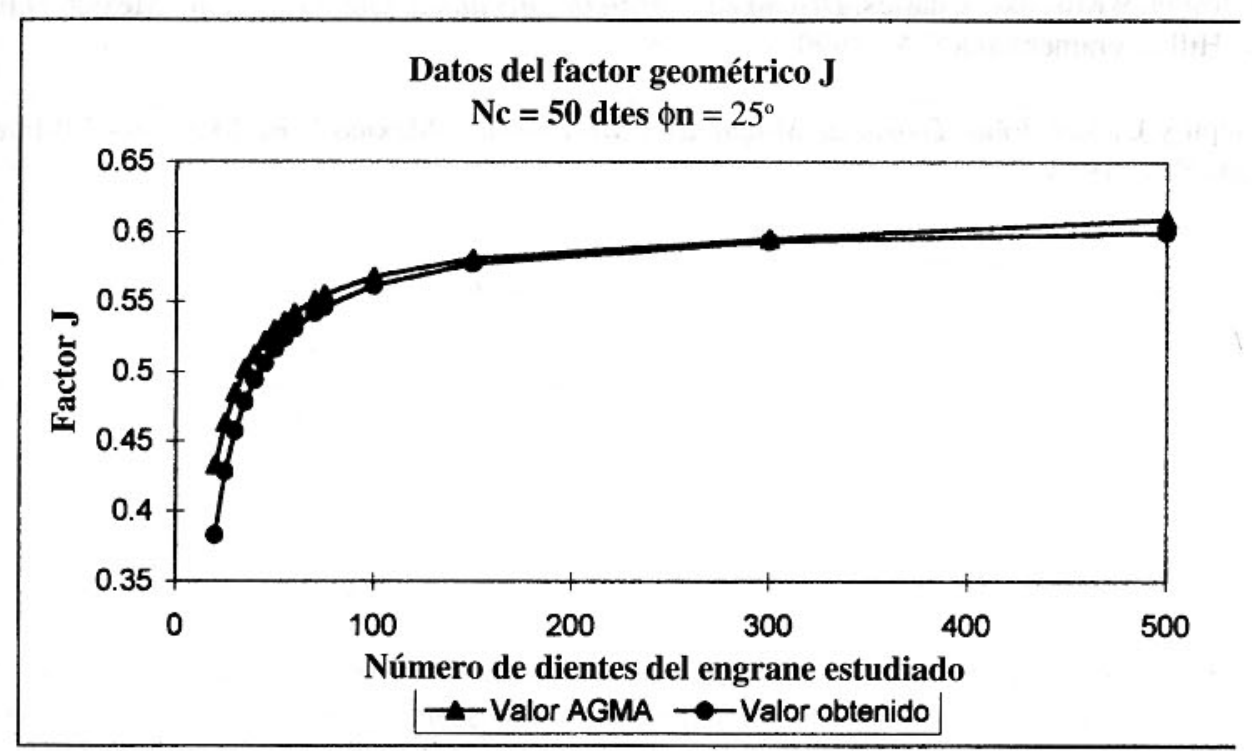

Fig. 11: Comparación del factor J para engranes rectos. 


\section{CONCLUSIONES}

El modelo matemático presentado permite calcular en forma analítica el factor $\mathrm{J}$ de cualquier engrane recto a partir de los números de dientes del par de engranes conectados y su ángulo de presión. Este modelo está constituido por un grupo numeroso de algoritmos matemáticos concatenados cuya solución se logra fácilmente con la ayuda de un programa de calculadora o de computadora.

Los valores del factor $\mathrm{J}$ calculados con el modelo fueron comparados con los valores gráficos del método AGMA, lográndose una aproximación asombrosa, lo cual valida la efectividad del mismo.

El modelo matemático desarrollado es fácil de programar y fue concebido para convertirse en una subrutina de un programa de cómputo mayor para el diseño de engranes.

\section{BIBLIOGRAFIA}

1. Burden, Richard y Faires, J. Douglas. Análisis Numérico. Tercera edición. México D.F.: Grupo Editorial Iberoamericana S.A., 1985.

2. Dudley, Darle W. Manual de engranes: Diseño, Manufactura y Aplicaciones de engranes. México D.F.: Compañía Editorial Continental S.A., 1973.

3. Shigley, Joseph y Mischke, Charles. Diseño en Ingeniería Mecánica. Cuarta edición. México D.F.: McGraw-Hill Interamericana S.A., 1985.

4. Shigley, Joseph y Mischke, Charles. Diseño en Ingeniería Mecánica. Quinta edición. México D.F.: McGraw-Hill Interamericana S.A., 1990.

5. Shigley, Joseph y Uicker, John. Teoría de Máquinas y Mecanismos. México D.F.: McGraw-Hill Interamericana S.A., 1983. 